\title{
Management of dengue haemorrhagic fever (DHF)
}

\author{
G N Lucas ${ }^{1}$ \\ Sri Lanka Journal of Child Health, 2000; 29: 107-8
}

(Key words: Dengue haemorrhagic fever, DHF, management)

The major pathophysiological abnormality seen in DHF is an acute increase in vascular permeability leading to plasma leakage followed by hypovolaemic shock if the loss of plasma is critical. A reduction of plasma volume exceeding $20 \%$ has been noted in severe cases. Supportive evidence for plasma leakage include serous effusion, haemoconcentration and hypoalbuminaemia. Hypovolaemic shock leads to tissue anoxia, metabolic acidosis and death if uncorrected. In the case of prolonged uncontrolled shock disseminated intra-vascular clotting (DIC) may cause clinical bleeding and may play an important part in the development of lethal shock. About one third of shock cases, mostly those with refractory shock, present with bleeding especially from the gastro-intestinal tract. In the majority of patients who die gastrointestinal haemorrhage is a constant finding at autopsy. Early recognition of shock and rapid volume replacement will usually prevent clinical DIC. Serial determination of platelet and haematocrit values are essential for early recognition and prevention of shock. The critical period in severe cases is the transition from the febrile to the afebrile phase which typically occurs after the third day of illness.

Fluids used for rapid volume expansion include the following:

- Ringer lactate (Hartmann solution)

- $5 \%$ dextrose diluted $1: 2$ or $1: 1$ in normal saline.

- Dextran 40

- Plasma

Rest in bed is advisable during the acute febrile phase of suspected DHF. Antipyreties and tepid sponging are required to keep the body temperature below $40^{\circ} \mathrm{C}$. Oral fluid and electrolyte therapy is recommended for patients with excessive sweating, vomiting or diarrhoea. A rise in haematocrit (HCT) value of more than $20 \%$ from baseline (or HCT > $40 \%$ if no baseline value is available) indicates significant plasma loss and a need for parenteral fluid

${ }^{1}$ Consultant Paediatrician, Lady Ridgeway Hospital, Colombo. therapy. The presence of a lamellar pleural effusion in a chest $\mathrm{x}$-ray taken in the right recumbent position has the same significance.

Serial haematocrit determinations every 4-6 hours and frequent recording of vital signs are recommended for adjusting the fluid replacement in order to assure adequate volume replacement and avoid over-transfusion. The fluid should be the minimum volume that is sufficient to maintain effective circulation during the period of leakage (2448 hours). Excessive replacement will cause respiratory distress (from pleural effusion), pulmonary congestion and generalized oedema.

\section{Treatment of dengue shock syndrome (DSS/ Grades 3 \& 4 DHF)}

DSS is a medical emergency that requires prompt and vigorous volume replacement therapy. The plasma loss should be immediately replaced with Hartmann solution or dextrose-saline at the rate of 10-20 $\mathrm{ml} / \mathrm{kg} / \mathrm{hr}$ or in the case of Grade 4 DHF as a bolus of $10 \mathrm{ml} / \mathrm{kg}$ (once or twice). If there is no improvement in the vital signs and the haematocrit values remain high, dextran 40 or plasma should be given at a rate of $10-20 \mathrm{ml} / \mathrm{kg} / \mathrm{hr}$. If there is no improvement in the vital signs despite declining haematocrit values, fresh whole-blood transfusion is indicated $(10 \mathrm{ml} / \mathrm{kg} / \mathrm{hr})$. When improvement is apparent, the rate of IV fluid administration should be reduced and adjusted one to two hourly, guided by haematocrit values and vital signs, for a period of 24-48 hours. IV fluid should be discontinued when the haematocrit reading drops to around $40 \%$ and vital signs are stable. A good urine flow indicates sufficient circulating volume. A return of appetite and diuresis are signs of recovery. In general, there is no need for fluid therapy for more than 48 hours after onset of leakage and/or shock. Reabsorption of extravasated plasma occurs over the next one or not significant bleeding. Insertion of an intragastric tube to determine concealed bleeding is hazardous and is two days and may cause hypervolaemia, heart failure and pulmonary oedema if more fluid is given. Oxygen therapy should be given to all patients with shock. Fresh frozen plasma is indicated when DIC causes recommended. 
Generally anti-coagulant therapy is not indicated for DIC. However, in patients with prolonged shock where metabolic acidosis has developed and in whom shock is refractory to treatment, the use of heparin may help to break the vicious cycle of shock and DIC before the stage of irreversibility is reached.

\section{Treatment of Grades 1 and 2 DHF}

It is recommended that IV fluid therapy be commenced with Hartmann solution or dextrosesaline at the rate of $6 \mathrm{ml} / \mathrm{kg} / \mathrm{hr}$. Once improvement occurs as shown by a falling haematocrit, stable blood pressure and a rising urine output, the rate is reduced to $5 \mathrm{ml} / \mathrm{kg} / \mathrm{hr}$, then to $3 \mathrm{ml} / \mathrm{kg} / \mathrm{hr}$ and discontinued after $24-48$ hours. If the vital signs or haematocrit worsens the rate is increased to $10-20$ $\mathrm{ml} / \mathrm{kg} / \mathrm{hr}$ and treatment is instituted as for DSS.

In Sri Lanka, in most hospitals, haematocrits can be done only once or at the most twice a day. Thus, if reliance is placed solely on the haematocrit, the initial fluid rate of $6 \mathrm{ml} / \mathrm{kg} / \mathrm{hr}$ will be reduced to $5 \mathrm{ml} / \mathrm{kg} / \mathrm{hr}$ only at the end of 12 hours and further reduced to 3 $\mathrm{ml} / \mathrm{kg} / \mathrm{hr}$ only at the end of 24 hours. At the end of 48 hours when the fluid is discontinued the child will thus have respiratory distress (from massive pleural effusion and ascites), pulmonary congestion and oedema. Thus, at LRH, I tend to rely more on the vital signs than on the haematocrit. I prefer to start with a fluid rate of $1-2 \mathrm{ml} / \mathrm{kg} / \mathrm{hr}$ and gradually increase the rate if the vital signs deteriorate. Over the last few years I have rarely had to exceed a rate of $3 \mathrm{ml} / \mathrm{kg} / \mathrm{hr}$ in the management of Grades 1 and 2 DHF.

\section{Source}

Nimmannitiya S. Management of Dengue and Dengue Haemorrhagic Fever. In Monograph on Dengue/ Dengue Haemorrhagic Fever, New Delhi. WHO regional publication SEARO 22, 1993; pp 5561.

\section{$\underline{\text { Editorial Comment }}$}

In the local setting, inappropriate and repeated investigations like Qualitative Antibody Tests rarely help in acute management of suspected cases of Dengue Fever. Extensive investigation during the first 2 days of the illness, apart perhaps from baseline PCV and platelet counts, are also not helpful. What is required in our scenario is continued and hawk-like vigilance coupled with judicious management. It is of paramount importance to carefully regulate fluid management as, in many cases, fatalities are suspected to be due to over-zealous treatment. Extensive experience in the management of dengue cases in the past, on the part of the medical personnel, seems to be an important factor associated with success in the management of these cases.

\section{B J C Perera} Joint editor 
